\title{
Fengxiang Color Protection Digital Protection in Shaanxi
}

\author{
Junyi Hou \\ Weinan Normal University, Weinan, Shaanxi, 714099
}

Keywords: Fengxiang; Shaanxi; digital storage; high simulation modeling

\begin{abstract}
In today's era of rapid development of digital information technology, the use of today's digital technology to protect the traditional hand-painted colorful products "Shaanxi Fengxiang colorful plastic" for the first batch of national non-material cultural heritage as: "Shaanxi Fengxiang colorful plastic" Folk art with a high cultural value. High-level simulation modeling of classic color plastic finishing archives, module analysis and establishment of a model database for digital storage and protection of color plastics are shown in this paper. In the later period, the model, the virtual image and the existing colorful art works are combined, and through digital interactive practice, on the basis of inheriting the essence of Fengxiang's colorful art, to enhance its artistic expression.
\end{abstract}

\section{Introduction}

As an ancient folk art culture in China, Shaanxi Fengxiang Color Plastics has a history of sedimentation for hundreds of years. It has Shaanxi culture and customs and local flavor. However, the most famous artists of the color sculptures are all old. Their unique production techniques, artistic expression methods, and knowledge and understanding of the color sculptures cannot be continued. They are looking for the artistic features and cultural essences that can produce and pass on the color sculptures. It is difficult for people to pass on the word, and the posterity of most artists choose to leave their hometowns, go to study, and seek other ways of life. Therefore, they must take advantage of digital means to participate in the protection and inheritance of the masters of art and inherit the national culture.

\section{The Status of Fengxiang's Digital Protection}

Shaanxi Fengxiang is located in the western part of the Guanzhong Plain, and is also one of the birthplaces of the 5,000-year-old civilized and historical pre-Qin dynasty in China. It records the historical records of the "Silk Road" during the Han and Tang dynasties. As a kind of traditional folk art handicraft carving in Shaanxi, Fengxiang Color Plastics carries the "remember" of a nation's original ecology and is called "mud goods" by the local people. It is also a "carrying goods" when it is a child. As early as in the Spring and Autumn Period and the Warring States period, people had unique insights and perceptions of color plasticity. They used colored plastic to do funerals and worships of Buddhist images and began to intervene in color plastics. From simple ceramic pottery to simple, colorful, traditional arts and crafts of different shapes, it has been handed down from generation to generation, filled with strong local flavor, ethnic flavor and local people's atmosphere.

At present, there are few researches on the digital protection of Fengxiang color plastics. Most researchers have proposed different research methods for digital protection: Gao Ximing's “Digital Protection of Intangible Cultural Heritage Resources in Henan Province” mainly uses digital information and multimedia technology. Modern software technology protects non-cultural heritage [4]; Wang Jianming proposed a method for the protection of intangible cultural heritage in the "Study on the Protection Strategies of Intangible Cultural Heritage Based on Digital Technology" and used three museums to protect cultural heritage materials to protect the inheritance of higher education. And cultural heritage, the development of tourism and cultural industries, so that people understand the cultural connotation. Previous researches on digital protection have mainly focused on the background, status, development, challenges faced by digitalized protection, digital storage, digital animation, comparison of digital protection at home and abroad, and the establishment of 
digital museums, libraries, and exhibition halls.

After a team interview, it was learned that $80 \%$ of local households in Fengxiang have been engaged in the production and management of colorful plastics, as the local residents have seen its value and interests. The scale of color printing has also rapidly increased, and the economy of Liuying Village has increased. It has also been improved. But in this process, there are also some problems in the development of color plastics, and we need to pay attention.

Today's colorful plastic production methods are more rigid, resulting in the inability to produce a large number of products. In the 1999 Kunming World Expo, because of its relatively small scale of production and relatively low output, it was unable to meet the needs of customers and missed a large sales order with Germany, so it was difficult to complete cooperation with larger manufacturers. Secondly, the mode of production is relatively decentralized, and the production of the self-employed households is the main reason. There is no centralized management system, which affects the overall image awareness and the workmanship is not precise and imperfect. This is not conducive to the expansion of production scale and the development of industrialization.

Local villagers lack awareness of protection of property rights in color plastic products. The image of product packaging also lacks sufficient creativity, lack of novelty, and how to establish a brand awareness that can attract buyers' desires, so that colorful models can be integrated into modern and diversified lives. These are all influences. The important factor in the development of color plastic. The production of colorful plastics is mainly based on human hand-making, so that there will be physical differences in the shaping of color plastics, and the lack of craftsmanship and technical quality design will have a huge impact on the shaping of color plastics and subsequent production, and most of the successors Both descendants and bachelors are imitations, copying, copying, and lack of innovation, which reduces the original value of color plastic.

\section{The Application of Digital Technology in Fengxiang Color Plastic Art}

Fengxiang Plastics uses the red, green, and yellow colors as its basic colors (a symbol of auspiciousness, as well as the meaning of celebration), and adds black and white (for sketching purposes, outlines mainly through auxiliary lines), plus five colors (white and blue). , black, red, yellow) to shape its physical beauty, and have a certain connection with China's yin and yang elements (gold, wood, water, fire, earth) and without losing the overall texture of color, the color of the cold, warm, saturated, color, The contrast is unique, simple and natural, giving people a beautiful enjoyment.

In terms of shaping the color sculptures, the figures are exaggerated and lively, with symmetry and balanced beauty. Each design has its own unique style, such as the Chinese Zodiac modelling, and there is a natural arrogance and momentum that Hongbo operates to explain the simplicity. Thought, this is also the artist's unique understanding of color sculpture.

In the depiction of appearance instruments, many painted patterns such as lilies, jellyfish flowers, green leaves, and wormwood are used to outline the image. The image that is created symbolizes nobleness, prosperity, grace, dignity, love, and profound nationality. Implications, the local artists pay attention to the smoothness and smoothness of the "smooth" lines in their delineation and shaping. They are organized in an orderly manner, enabling the color sculptures to be both in the external portrayal and the intrinsic emotional coloring of the creators. Good integration.

Fengxiang Color Plastics is a combination of both carving and painting art. It is color-touching, coloring and symbolic. It has roughly 170 types and can be divided into three types: First, it's different in size, mainly in entertainment and play. In color, there are many types of animals; in the second, there are hanging objects worn on the wall or worn on the body; and third, the characters are mainly shaped (mostly historical figures). Most of these colorful arts are static, and it is not easy to actively communicate to the audience art information. This will cause a considerable part of the art of color sculpture to be ignored.

Compared with traditional art, digital technology has increased the interaction between the two sides (breaking the traditional single mode of communication, can interact with each other in a two-way way, understanding the voice of the audience), virtuality (virtual environment scenes, 
without human contact learning), and multi-media ( From a technical and artistic point of view, it can be communicated through images, animations, later stages, and the Internet in many ways, uncontrolled (not affected by any factors such as time and place, or insufficient storage). The use of digital technology to organize and reposition the artistic characteristics of colorful plastics, use modern digital technologies to protect the colorful plastic cultural resources, carry out digital research, and then incorporate digital technologies to participate in the process of art, technological innovation, and cultural heritage, which is beneficial to The research and plasticity in the later period can make the colorful plastics closely linked with our lives and will always be closely related to the sustainable development.

Combining the virtual modeling image with the existing colorful art objects and using the sound and optoelectronics (here, the sound and light is not realized by adding sound, light and darkness, and light perception in the artistic creation, but in the physical sense of technology display and reality. Realized digital technology to realize multiple dynamic display modes, demonstrating three-dimensional true color (using advanced texture patterns to analyze objects, reconstructing three-dimensional data, and automatically and precisely combining texture patterns in three-dimensional coordinates. This will not distort the color of the original object itself, and the resolution of the produced model will be relatively high.) The static dynamic image will be the modelling and illusion of the color model (the model will be added to the camera and the scene lighting to adjust the mutual position between them. The image projection obtained after rendering is combined with the theme model landscape of the scene box to achieve realistic display. A holographic projection technology and a three-dimensional scanning technology are used to create a virtual model. A multi-angle shooting and image imaging are used to create a high-precision image. Digitized archives, centralizing the well-established models to create 3D digital museums Such colorful combination of art through digital technology features will be shaped by a very good, and finally more intuitive 3D digital technology show in front of an audience.

The digital protection of Fengxiang Caisu is of high value, and it is also conducive to the development of the folk art industry. As an ethnic industry development chain, Fengxiang Caisu has invested less than the input of other industries at a considerable level, which is conducive to the development of the industry. Secondly, as an intangible cultural heritage, Fengxiang Color Plastics displays the cultural heritage of a nation and the importance that a country attaches to cultural protection, and establishes a library of colorful plastic data, which can reduce the various causes of color plastic in the manufacturing process. damage. Again, through digital methods, it is very convenient for the graphic audiovisual and digital technology information to be well transformed between the two, to facilitate subsequent transmission and invocation, to demonstrate the unique artistic charm of the color plastics, and also to last for a long time. The protection of sex provides better and convenient conditions. Finally, digital protection can also increase the conservation of conservation and avoid the use of traditional resources to repair large amounts of natural resources (this is mainly consumed by the use of materials in the production process), protecting both cultural heritage and indirect Sexual trampling and waste of resources.

\section{Fengxiang Colorful Digital Protection}

Digital file storage: The use of digital technology to text, images, video and other resources, through the establishment of network file data on the cultural relics, physical structure scanning, photography, processing and then stored, to achieve its long-term storage and preservation.

Digital file storage, mainly through the use of digital technology to obtain colorful plastic processing information (a comprehensive investigation of excavation, sorting, collection, classification, color plastic filing and post-integrated management) This is a relatively new type of protection method, the traditional protection model Editing, converting to a digital format for processing, sorting and processing well-processed information technologies into digital media such as computers, external memories, and digital memories, so that protection can be completed. Traditional color protection is newly protected and formed. A new way of protection, using digital storage protection, not only takes up a lot of storage space, but also can be a flexible digital model 
conversion, easy to carry, edit, move, add and delete, simple and convenient delivery, people do not have to be subject to conditions Can observe and appreciate, will not be affected by too many economic interests, so as to choose a higher practical production and production. Through the informationization of the Internet, this folk culture is protected and disseminated as a whole, which is conducive to the advancement of non-material culture.

High simulation modeling: In order to study and analyze a certain object or phenomenon, establish a model or graphic image with a high degree of similarity with the real-world system, which can be simply called true reproduction, and then use this model for experimentation or research ( Digital technology is used to recreate graphics, and innovative designs create more appealing masterpieces, called high-fidelity modeling.

Through digital shooting, three-dimensional scanning (which is a non-contact optical scanning, recording the three-dimensional coordinates of colorful images to generate image data and color and other related information generated), holographic shooting and other digital technologies establish a virtual model library. Through optical scanning and holographic imaging processing technology, the model is transferred to a digital converter to be converted into an analog digital signal and then transmitted. The common features and color patterns of the colorful plastics are extracted, and the classic works are virtually repaired and archived, so that they can be viewed directly. The virtual products, people adopt 3D animation technology, TV movies or the Internet to reproduce the colorful model display, scene construction, action binding and character generation, while protecting the traditional folk art, avoiding the development of color Obstruction in the process of inheritance.

Classical colorful plastics and endangered colorful paints must be comprehensively compared in various aspects during the production process, collecting the data and related data needed for creation, and performing high-simulation modeling (in order to achieve the cooperation between the elements of the point and the surface during the production process. From the perspective of observation, creation and visualization of virtual restoration of color and plasticity, a graphical database is established to provide a new way for the generation of three-dimensional scenes, and to reproduce the damaged or impaired colorful national cultures, regardless of the previous work (collection, arrangement, arrangement). , record) or later (processing, simulation, processing, preservation) can better record our cultural heritage.

A good piece of work is inseparable from a good designer. The design and color of the colorful plastic design and appearance are inseparable from the opinions of those artists. How to realize the meticulous design of colorful plastic? This requires the establishment of a unique design concept to achieve a consensus on the combination of external beauty and internal beauty without destroying the essence of the color plasticity (the external image and shape of the color plastic itself is not modified and artistically processed). Design a set of software programs (after initial modeling and overall aesthetic design of color plastics, conduct dynamic programming modeling, make color plastics have "soul", combine 3D animation software, virtual simulation animation (using virtual reality technology to draw Scene model) and computer programming, digital scenes through digital media (digital technology, network information technology, production technology) show, and then map its color, post-render synthesis, so that you can show the vivid color of life, plus Words, sounds, and images are explained to maximize the sharing and use of color plastics.

The design and production are firstly digital induction, classifying and classifying the categories of the colorful plastics (design concepts and solutions), building models using 3D design software to analyze the modules, establishing a graphical database or using 3D scanning and holographic imaging technologies to realize images Of course, the binding of skeletal animation can be carried out, programming can be performed using programming techniques, and the model created can be dynamically displayed. For example, the Zodiac, the model is given life, and a three-dimensional animated short film is produced. The author can then Adding your own design elements while creating an animation is not only the protection of the color plastics, but also the development of another industry. Finally, the designed physical object will be displayed in a centralized manner to realize the virtual restoration of the model, the later processing of the produced model, and the 
rendering of the output model by the special effect, so that a true reproduction is formed.

Fengxiang's colorful, artistic and aesthetically pleasing, regionally-oriented cultural features have also undergone economic changes in Fengxiang County, and the local architectural environment of Caisu Village has undergone tremendous changes, replacing traditional architectural models with new rural and new buildings, but in the process In the past, the living environment of the colorful plastics has been affected, losing the characteristics of traditional folk art and decompressing the regional characteristics of the colorful plastics. Therefore, the establishment of a folk art museum for the protection of color plastics must be based on the true value of the colorful plastics and the living environment of the local villagers. In order to better communicate the essential features of the colorful plastic arts, it is necessary to maintain the artistic connotation and cultural value of the traditional arts. The establishment of folk art museums Compared with digital museums, investment in small, low-cost, small-scale construction sites do not occupy the existing sites to make minor changes and adjustments to facilitate future work.

The establishment of a natural and ecological folk art museum is based on the living environment of the residence of Liuying Village. The local villagers, as the carrier of the folk art museum, have concentrated on the establishment of residential and residential integrated architectural style folk art museums (or the entire Liuying Village). And the surrounding environment as an integral part of the original ecological folk art museum), free from the defects of individuality and independence, and create a good place for colorful plastics production craftsmanship and construction environment to facilitate centralized production, processing, production, and post-baking processing. It not only alleviates the disadvantages of self-employment, but also helps Sixying Village establish its own brand awareness, unify production centralized management, implement the production goals of "one village, one point, one product", and display the unique charm of colorful folk culture, not only The display of products is to display the production process and historical transformation of folk art culture, highlighting the integration of the connotation of the art of color sculpture and real life.

Digital protection of the color plastics can also be entered into the campus. Students who have higher appreciation and pursuit of color plastics can use the knowledge they have learned and their knowledge and understanding of the color plastics to create two-dimensional animated cartoons and join the students. The design elements and visual performances present the images of their hearts. After being guided by professionals to students, they are digitally integrated with real-life rendering techniques. They use digital technology for later synthesis and processing, so that they can make virtual models virtual. In the world to achieve real-world artistic features and colors. This will not only achieve the protection of color plastic, but also create a colorful charm, but also brings a new way for the digital protection of color.

Here, we can add interactive design to the reproduction of colorful plastics, break the traditional art's thinking mode, and eventually do a good job of the colorful plastic design. Through Internet technology, we can establish centralized display on the Internet. Students and netizens can search for related designs of colorful plastics through the establishment of the public The publicly-developed communication and dialogue platform can add its own style and design concept to the well-established model and design of the model, and better reflect the art appreciation of the public. Or it is to program the color plastic design. Students and netizens only need to open the computer with a click of the mouse, and they can display the visual impact effect of the colorful plastic in various aspects such as plane, panorama, and three-dimensional space without limitation. (For example, the "sheep" in the zodiac, the viewer can drag or turn the mouse, and then the sheep's eyes will turn together with the viewer's drag or turn the direction of the mouse to make the sheep walk or do various Movement requires only inputting instructions, and the sheep can complete walking and various kinds of sports.) Realize the interaction between people and objects, improve the cooperation and development of interactions in the technical field, browse anywhere, anytime, even if the follow-up needs to be modified will not be affected. Limitations and impact of preservation incompleteness.

Digital file storage provides the foundation for Fengxiang's digital protection. High simulation 
modeling provides a fulcrum for the digital protection of Fengxiang. The digital design software provides space for the digital protection of Fengxiang, and the digitalized color folk museum is Fengxiang's. Digital protection has built a platform, and digital interactive practice has injected new vitality into the protection of Fengxiang.

\section{Conclusion}

In the business environment, today's Shaanxi Fengxiang color plastic production is just to meet the market's needs, with sales as the production of production and customer demand as the main means of production, which makes many traditional products disappear and the traditional color plastic technology is missing. It also caused Fengxiang Colors to gradually lose its original natural beauty. This has caused people's perception of color sculptures to be affected, and also limited the transmission of intangible cultural heritage in China. Therefore, digital technology is used to complete the protection of color sculptures so as not to lose color space. With the charm of the original ecology. As an ancient folk art form in Shaanxi, Fengxiang Caisu serves as the precious cultural "gene" of the Chinese nation and maintains the cultural and ecological prosperity. It will surely be accompanied by a higher level of protection for digital technology and arts, and it will be continuously broken through. It will also be better strengthened.

\section{Acknowledgment}

Project Name: Visual Simulation Platform Design Based on Digital Modeling Technology Project ID: 17JMR34

\section{References}

[1] Yang Xiaoyan, Feng Dong, Zhang Shuhua, Zhang Zhihua. The Existence and Development of Shaanxi Folk Art from Fengxiang Clay[J]. Northwest Industry Journal of Chinese Universities (Social Science Edition), 2007, (04): 25-28.

[2] Xue Rui. The Chifeng intangible cultural heritage [J]. Practice (ideological theory version), 2011, (04): 54.

[3] Li Yanan. Research on the application characteristics and aesthetic connotation of Fengxiang's clay color[D]. Xi'an: Xi'an Academy of Fine Arts, 2009.

[4] Ni Tong. Research and development of intangible cultural heritage system based on JSP [D]. Zhengzhou: Zhengzhou University, 2012.

[5] Wang Jianming, Wang Shubin, Chen Shipin. Research on the Protection Strategy of Intangible Cultural Heritage Based on Digital Technology[J]. Software Herald, 2011, (08):49-51. 